\title{
Accurate measurement of a fission chamber efficiency using the prompt fission neutron method
}

\author{
Ludovic Mathieu $^{1, \text { a }}$, Mourad Aïche ${ }^{1}$, Grégoire Kessedjian ${ }^{2}$, Serge Czajkowski ${ }^{1}$, Beatriz Jurado ${ }^{1}$, Paola Marini ${ }^{1}$, \\ and Igor Tsekhanovich ${ }^{1}$ \\ ${ }^{1}$ CENBG, CNRS/IN2P3-Université de Bordeaux, Chemin du Solarium, BP. 120, 33175 Gradignan, France \\ 2 Laboratoire de Physique Subatomique et de Cosmologie, CNRS/IN2P3, Univ. Joseph Fourier, INPG, 53 avenue des Martyrs, \\ 38026 Grenoble Cedex, France
}

\begin{abstract}
Fission Chambers (FC) are often used to determine fission cross sections and to measure the neutron beam flux via standard neutron-induced fission reactions. Thus, the fission detection efficiency is a key parameter. Several methods exist to determine this efficiency, with a final accuracy not better than $1 \%$. The detection of prompt fission neutrons allows events related to the fission process to be tagged, and enables the efficiency to be inferred with accuracy of the order of few $0.1 \%$. This method is very robust since it is independent in first order to several factors like geometry, used materials or neutron contour selection. To obtain high accuracy, few corrections have still to be taken into account. In particular, the neutron detectors have to cover several detection angles. In addition, the background contribution of neutrons from cosmic rays or from an accelerator has to be removed. Several experiments based on the use of a ${ }^{252} \mathrm{Cf}$ source are presented to describe all these points.
\end{abstract}

\section{Introduction}

Ionization chambers using fissile material (called Fission Chambers (FC)) are very simple and versatile devices [1] used in several fields in nuclear physics, applications in nuclear industry or nuclear energy research [2-4]. In the field of nuclear data measurements, FCs are used to measure fission cross sections $[5,6]$ and as neutron flux monitors $[7,8]$. They are also used to tag fission events in order to study fission-related phenomena $[9,10]$ or to reject fission events to study hardly observable phenomena [11].

FCs generally have a very high efficiency, only limited by the self-absorption of the Fission Fragments (FF) in the sample when they are emitted at very large angles with respect to the target normal $\left(\sim 90^{\circ}\right)$. For many applications, the knowledge of the efficiency is a key parameter to obtain accurate results.

Moreover, when one is interested in a weak phenomenon whose signature is hidden by the fission process (for instance the radiative capture), the clean subtraction of fission events is crucial. In particular, the fission events undetected by the FC have to be estimated and also subtracted. As the statistics of the investigated process is weak when compared to the one of the fission process (due to differences in cross sections or secondary particle emission multiplicities), the uncertainty on the result is proportional to the efficiency uncertainty but the proportionality coefficient may be quite large. For radiative capture cross section of a fissile isotope, this coefficient ranges from 5 to 30 in the thermal or epithermal energy range. In such cases, the very accurate knowledge of the FC efficiency is of paramount importance.

a e-mail: mathieu@cenbg.in2p3.fr

\section{Efficiency measurement methods}

\subsection{Usual methods}

There are different ways to measure the FC efficiency, depending on the spontaneous-fission half-life of the sample, the knowledge of its fission cross section or the detector used.

The simplest case is when the nucleus of interest fissions spontaneously (like ${ }^{252} \mathrm{Cf}$ ). Then the fission rate depends only on the amount of material, which is easily obtained via alpha spectrometry. The main uncertainty sources of this method are the solid angle of the alpha spectrometer and the dead-time correction of the fission measurement. For certain nuclei, the spontaneous fission yield $\left(\mathrm{y}_{\mathrm{SF}}\right)$ may also be a significant source of uncertainty (for instance $\mathrm{y}_{\mathrm{SF}}$ of ${ }^{240} \mathrm{Pu}$ has an uncertainty of 3.5\% [12]). For ${ }^{252} \mathrm{Cf}$, an uncertainty of $1 \%$ for the fission efficiency can be achieved.

If the nucleus does not fission spontaneously another solution is to use a Frisch-gridded FC. This device allows one to estimate the emission angle of the FF. If the fissionfragment angular distribution is known, the missing FF at high angle can be inferred [13]. The uncertainty of this method can be as low as 1\% [14].

In the cases where no Frisch-grid is used, it is much more complex to measure the fission efficiency. It can be calibrated thanks to a reference Fission Chamber (e.g. ${ }^{235} \mathrm{U}(\mathrm{n}, \mathrm{f})$ ) or Ionisation Chamber (e.g. ${ }^{10} \mathrm{~B}(\mathrm{n}, \alpha)$ or $\left.{ }^{6} \operatorname{Li}(n, t) \ldots\right)$. It then depends on many parameters $(\alpha$-spectrometry efficiency, decay constant, fission cross section...) for the studied and reference material, as well as the dead time correction and the efficiency of the reference fission or ionization chamber. In the end, 
the accuracy of the FC efficiency is not better than few percents with this method.

The fission detection efficiency can also be estimated by analytic calculations, taking into account the thickness of the deposit and the FF anisotropy [15]. The uncertainty of this method is again of the order of $1 \%$ [16].

Simulation can also be performed with Monte Carlo codes. Nowadays, such simulations do not have the required quality to be able to give an accurate value of the fission efficiency.

\subsection{Prompt fission neutron method}

A much simpler and more accurate method relies on using the prompt neutrons emitted by the FFs [17]. A very convenient way of detecting fission neutrons is with scintillators. In particular, $\mathrm{C}_{6} \mathrm{D}_{6}$ allow neutrons and $\gamma$-rays to be disentangled via Pulse-Shape Discrimination (PSD). These discriminated neutrons can then be detected in coincidence with a FF, and the FC efficiency $\varepsilon_{F C}$ is then given by the simple equation:

$$
\varepsilon_{\mathrm{FC}} \mathcal{D} \mathrm{N}_{\text {coinc-n\&FF }} / \mathrm{N}_{\text {single-n }}
$$

Where: $N_{\text {coinc }-n \& F F}$ is the number of neutrons detected in coincidence with a FF, and $N_{\text {single- } n}$ is the total number of detected neutrons.

Equation (1) remains valid, no matter how many neutrons where emitted by the fission process, or how many neutrons were detected at the same time by other scintillators. In addition, as FC efficiency is close to $100 \%$, the two terms of Eq. (1) are strongly correlated, greatly reducing the statistical error.

\subsection{Robustness of the method}

Due to the ratio expressed in Eq. (1), this method is very simple (no dead time correction needed, since dead time affects both terms in the same way), very stable in time and very robust against a lot of experimental parameters, such as the neutron contour used, the detector accurate location, or the materials used. In order to investigate this method, we carried out measurements with a ${ }^{252} \mathrm{Cf}$ source placed in a parallel-plate fission chamber and surrounded by several $\mathrm{C}_{6} \mathrm{D}_{6}$ detectors located at $90^{\circ}$ with respect to the target normal. Figure 1 presents the FC efficiency measured using two $\mathrm{C}_{6} \mathrm{D}_{6}$ detectors, which remained in the same configuration during all the test campaign. As can be seen, the measured efficiency is very stable over a period of more than 10 days: the results obtained with each detector are constant within $0.05 \%$, and the discrepancy between the two sets of data is of the order of $0.05 \%$.

In addition, a configuration has been tested with a $5 \mathrm{~cm}$-thick lead brick placed between the FC and a $\mathrm{C}_{6} \mathrm{D}_{6}$ detector. Once corrected from the background (see Sect. 4), the measured efficiency is in agreement with other measurements, with a high statistical uncertainty. This proves that materials located between the FC and the neutron detectors, even large thicknesses and high- $Z$ materials, do not introduce any bias in the method.

The method relies on the selection of neutrons in the PSD spectrum. Nevertheless the chosen neutron contour has nearly no impact on the measured efficiency, as can be seen in Figs. 2 and 3. Figure 2 presents several different neutron contours used, including two small

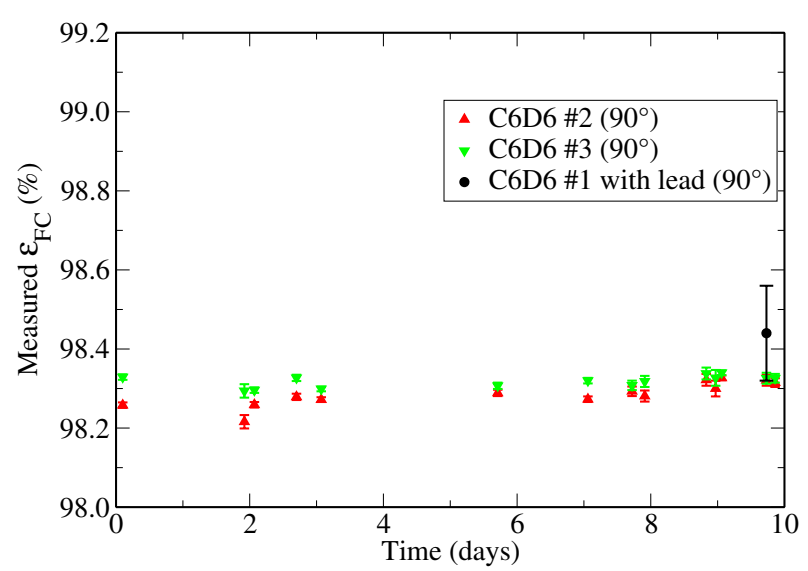

Figure 1. Time evolution of the measured efficiency of a ${ }^{252} \mathrm{Cf}$ fission chamber obtained with prompt neutrons detected in two $\mathrm{C}_{6} \mathrm{D}_{6}$ detectors. The efficiency measured in a configuration with a lead brick is indicated by the full black circle at $\mathrm{t} \sim 10$ days. The scale is chosen for comparison purpose with Figs. 3 and 4.

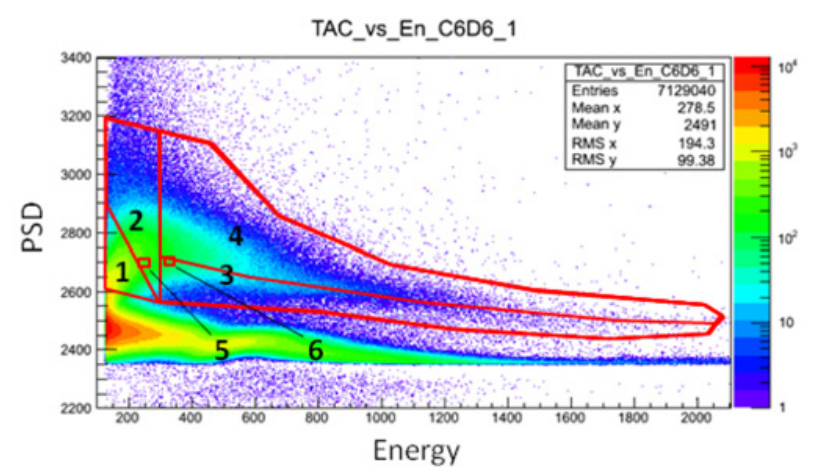

Figure 2. Pulse Shape Discrimination spectrum of neutron and $\gamma$-rays detected in $\mathrm{C}_{6} \mathrm{D}_{6}$ detectors. Different neutron contours are shown: (\#1) a very large one, (\#2) same as \#1 without the lowerleft corner, (\#3) same as \#2 without the low energy part, (\#4) same as \#3 without the lower half of the neutron banana, (\#5 and \#6) two very small contours.

contours selecting only very small parts of the detected neutrons. Figure 3 shows the measured efficiency for each of these contours. The results obtained are consistent whatever the contour used. Even the small contours give a correct efficiency, in spite of an increased statistical uncertainty. The efficiency can be measured using any fraction of the detected neutrons. Thus, the prompt fission neutron method can be used even if the neutron-gamma discrimination is not very good, as long as the neutron contour contains only neutrons (see Sect. 4).

\section{Influence of the neutron detector position}

In first order, the method does not require the knowledge of the angle, distance and dimension of the neutron detectors. These assumptions are not true in second order, although this is mainly ignored when this method is used [17]. Especially, a bias can be observed as a function of the neutron detector angle, as shown in Fig. 4. For this experiment, several measurements were carried out changing the angle of two $\mathrm{C}_{6} \mathrm{D}_{6}$ detectors. This means that the measured FC depends on the position of the neutron 


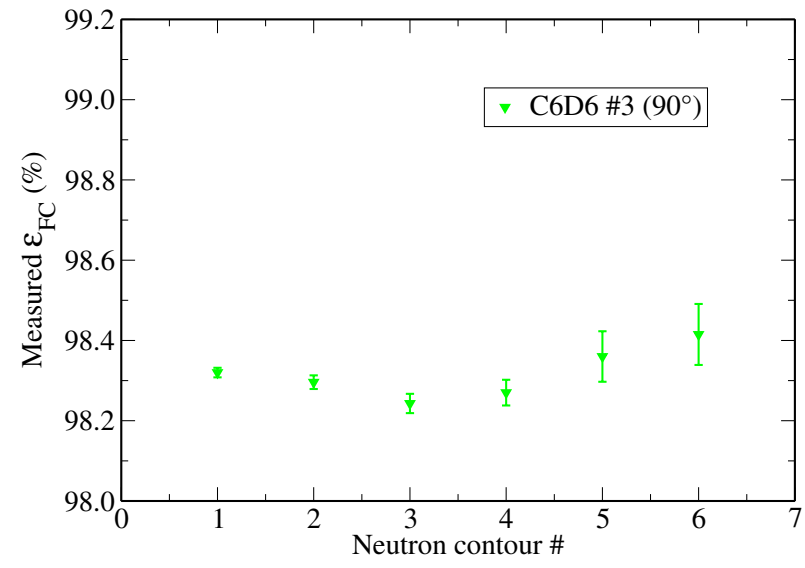

Figure 3. Measured efficiency for the neutron contour indicated in Fig. 2. The scale is chosen for comparison purpose with Figs. 1 and 4.

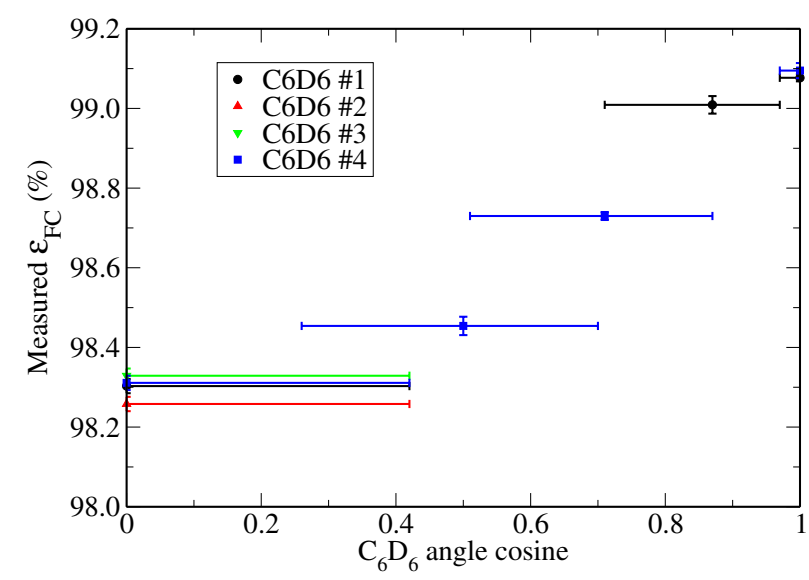

Figure 4. Measured efficiency for different neutron detector angles. The error bar in cosine represents the angular aperture of the front face of the $\mathrm{C}_{6} \mathrm{D}_{6}$. The scale is chosen for comparison purpose with Figs. 1 and 3.

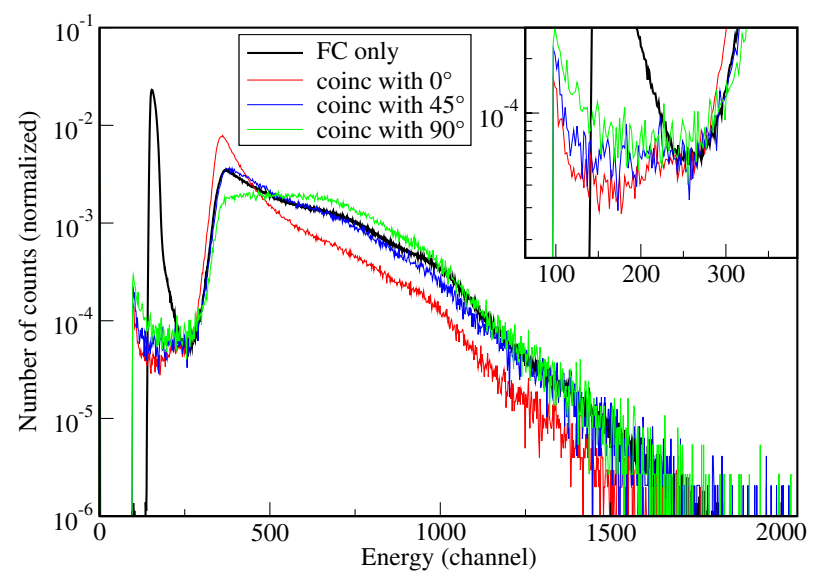

Figure 5. FC pulse-height spectra measured without coincidence (FC only, thick line), or in coincidence with neutrons detected in the $\mathrm{C}_{6} \mathrm{D}_{6}$ detectors located at various angles (thin lines). The alpha peak is present at low energy on the "FC only" spectrum.

detectors. This evolution can be understood by looking at the FC pulse-height spectra obtained in coincidence with the prompt neutron (Fig. 5).

The spectrum "FC only" has the standard shape of narrow parallel FCs: the peak due to alpha particles is visible at low energy (below channel 200), whereas the two FF peaks are barely visible around channel 1000 because of the very poor energy resolution. A FF emitted at $0^{\circ}$ with respect to the target normal loses only a limited amount of energy in the gas before hitting the opposite electrode (step increase of the spectrum near channel 300). As the FF emission angle increases, so does the energy loss in the gas (broad peak until channel 1500). For a FF emitted at grazing angle, a variable part of its energy may be lost in the sample itself, and the energy deposited in the gas can be as low as 0 . Thus, the threshold used to cut the alpha contribution also removes some FFs emitted at grazing angles.

Figure 5 also shows that the FF spectrum obtained in coincidence with a neutron detector depends on the angle of this detector: the spectrum is more peaked (more FF emitted at $0^{\circ}$ ) when in coincidence with detectors at $0^{\circ}$, and more flat (more FF emitted at high angle) when in coincidence with detectors at $90^{\circ}$. This is due to a kinematic effect of the FF velocity on the neutron momentum. This effect has also been confirmed by dedicated simulations, taking into account the emission of neutrons by the moving FF. As can be seen on the spectra, the FF part below the threshold is lower when in coincidence with a detector at $0^{\circ}$.

Because of this bias, none of the points of Fig. 4 represents the real efficiency of the chamber. The real efficiency can be inferred by integrating the measured efficiency over all the cosine bins. The aim of this procedure is to mimic the result of a $4 \pi$ detector surrounding the FC, and not to take into account the fission anisotropy. Indeed, the results already include this effect and no additional procedure is needed for that.

We obtain here a real efficiency of $(98.6 \pm 0.1) \%$. This result confirms the excellent performance of the prompt fission neutron method to infer a FC efficiency, at least for a thin ${ }^{252} \mathrm{Cf}$ source.

The good accuracy of this result is partly due to the fact that the efficiency spread is lower than $1 \%$ between measurements at $0^{\circ}$ and $90^{\circ}$. This will not be the case if the FC threshold is high (for instance at channel 500). Then, efficiency spread up to $30 \%$ between measurements at $0^{\circ}$ and $90^{\circ}$ has been observed. Under such condition, the average value cannot be accurate anymore. This implies that the prompt fission neutron method cannot be used if the FC threshold is high, i.e for highly radioactive samples where the $\alpha$ peak overlaps significantly with the FF peak.

\section{Influence of parasitic neutrons}

This method is also quite sensitive to parasitic events in the neutron contour. These parasitic events may be cosmic neutrons, fast neutrons coming from the beam, or wrongly discriminated $\gamma$-rays. As such events are not related to a fission process in the FC, they will reduce the measured efficiency according to Eq. (1). An experiment was carried out by placing the neutron detectors at different distances from the FC. The prompt fission neutrons are greatly reduced contrary to the background neutrons. As can be seen in Fig. 6, the measured efficiency drops strongly for large distances, for measurement at $0^{\circ}$ or at $90^{\circ}$. A correction can be applied to reduce this issue. By requesting a $\gamma$-ray detection in coincidence with the 


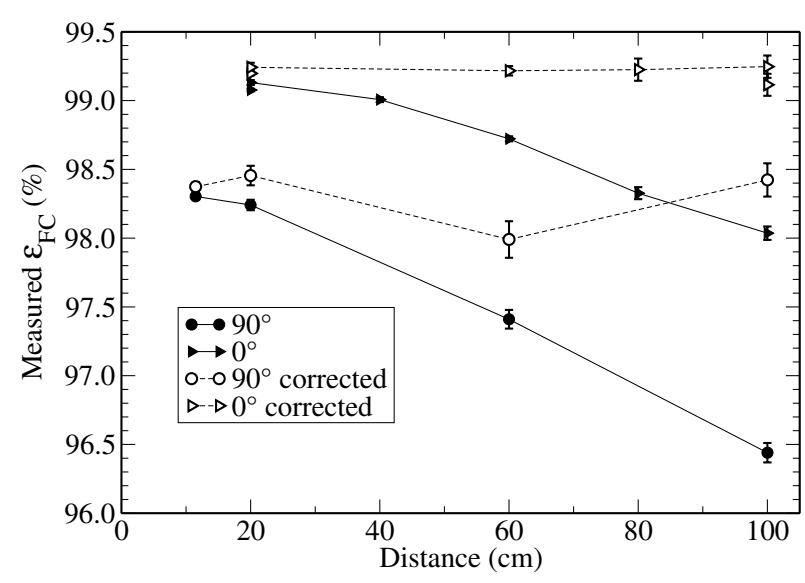

Figure 6. Measured efficiency with $\mathrm{C}_{6} \mathrm{D}_{6} \# 1$ placed at different distances either at $0^{\circ}$ or at $90^{\circ}$. Open symbols are for corrected data. Lines are here to guide the eyes.

prompt fission neutron, one will greatly suppress the parasitic events. Equation (1) then becomes:

$$
\varepsilon_{\mathrm{FC}} \mathcal{D} \mathrm{N}_{\text {coinc-n\&FF\& }} / \mathrm{N}_{\text {coinc-n\& } \gamma}
$$

As can be seen in Fig. 6, the "corrected" efficiency becomes more or less independent of the neutron detector distance. Nevertheless, this solution cannot overcome a too high amount of parasitic events. It implies that this method cannot be applied if:

- the signal-to-background ratio is too low,

- fast neutrons (produced by an accelerator) can be directly detected by the scintillator,

- a very intense $\gamma$-ray flux prevents completely the discrimination between neutrons and $\gamma$-rays.

\section{Conclusions}

The detection of prompt fission neutrons in coincidence or not with a Fission Fragment in the Fission Chamber enables an accurate value of the fission detection efficiency to be obtained. It has been shown that this method is very robust, since it is independent in first order to several factors like geometry, used materials or neutron contour.
Nevertheless, a small angular dependency has to be taken into account by detecting prompt neutrons at different angles with respect to the target normal. If the neutron background can be neglected, an efficiency accuracy of the order of $0.1 \%$ can be obtained for a thin source like ${ }^{252} \mathrm{Cf}$. If the neutron background is not negligible, an additional coincidence with a fission $\gamma$-ray may be implemented, at the price of lower statistics.

This work is supported by the European Commission within the 7th Framework Program through CHANDA (Project No. 605203).

\section{References}

[1] G.F. Knoll, Radiation Detection and Measurement $4^{\text {th }}$ Edition. Wiley (2011)

[2] IAEA (International Atomic Energy Agency)

[3] B. Geslot et al., Proceeding of ANIMMA Conference, Marseille, France (2009)

[4] J. Yang, Plasma Sci. and Tech. 7(3) (2005)

[5] S.A. Wender et al., Nucl. Instr. Meth. A 336, 226 (1993)

[6] M. Calviani et al., Nucl. Instr. and Meth. A 594, 220 (2008)

[7] P. Filliatre et al., Nucl. Instr. and Meth. A 593, 510 (2008)

[8] R. Nolte at al., Metrologia 48, 274 (2011)

[9] R. Billnert et al., Phys. Rev. C 87, 024601 (2013)

[10] A. Chatillon et al., Phys. Rev. C 89, 014611 (2014)

[11] I. Companis et al., Proceeding of CNR*11, Prague, Czech Republic (2011)

[12] Evaluated and Compiled Nuclear Structure Data http: //www.nndc. bnl.gov/ensdf

[13] C. Budtz-Jorgensen, H.-H. Knitter and G. Bortels, Nucl. Instr. and Meth. A 236, 630 (1985)

[14] P. Salvador, PhD thesis, Universitat Politècnica Da Catalunya Barcelonatech (in English)

[15] G.W. Carlson, Nucl. Instr. and Meth. A 119, 97 (1974)

[16] P. Salvador et al., Phys. Rev. C 92, 014620 (2015)

[17] V. Doolin et al., Nucl. Instr. and Meth 105, 2, 277 (1972) 